\title{
Das Mädchen und ihr Liebhaber. Pragmatik als motivierender Faktor von Sexuskongruenz
}

\begin{abstract}
Der vorliegende Beitrag beschäftigt sich mit den Einflussfaktoren, die die Auswahl der Kongruenzformen hybrider Nomina steuern. Es soll gezeigt werden, dass die Wahl dieser Kongruenzformen durch grammatische Faktoren - Typ des Pronomens und Distanz des Pronomens zum antezedenten Nomen - nur teilweise erklärt werden kann und dass außersprachliche Faktoren ebenso zur Formentscheidung beitragen. Aus diesem Grund wird in diesem Beitrag der Versuch einer Perspektiverweiterung unternommen, indem sowohl Merkmale aus Morphologie und Syntax als auch aus der Pragmatik zur Erklärung des Phänomens berücksichtigt werden. Es soll gezeigt werden, dass die kongruierenden Formen durch ein Interagieren der unterschiedlichen sprachlichen Ebenen gesteuert werden. Zu diesem Zweck wird zunächst der Untersuchungsgegenstand - die hybriden Nomina und deren Kongruenzformen - dargelegt und ein Überblick über die bisherige Forschung gegeben. Die auf dieser Grundlage formulierten Annahmen werden schließlich in einer Korpusstudie überprüft. Auf der Grundlage der Ergebnisse kann schließlich ein prototypischer Kontext für das Auftreten sexuskongruenter Formen abgeleitet werden, mit dessen Hilfe probabilistische Aussagen über das Kongruenzverhalten möglich sind.
\end{abstract}

\section{Gegenstand und Forschungsüberblick}

Die deutsche Syntax verlangt für jede Nominalphrase (NP) die Zuweisung eines Genuswerts. Bei Menschenbezeichnungen besteht in der Regel eine Korrelation zwischen Genus und Sexus, das bedeutet, dass in diesem Teil des Genussystems eine semantische Motiviertheit vorliegt. Ein viel diskutierter Aspekt ist daher der Zusammenhang von Genus und Sexus (vgl. Klann-Delius 2005; Leiss 1994, 2009). Auch bei der Pronominalisierung der Menschenbezeichnungen besteht zwischen Pronomen und Antezedens eine Genus-Sexus-Kongruenz (vgl. Köpcke \& Zubin 2005).

Julia Hübner, Institut für Deutsche und Niederländische Philologie, Freie Universität Berlin 
Die kontrollierenden Nomina in den beiden folgenden Beispielen (Frau bzw. Mann) übertragen ihren Genuswert (in diesem Fall F oder M) auf alle vorhandenen adnominalen sowie pronominalen Ziele.

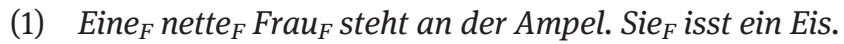

(2) Ein $_{M}$ netter $_{M}$ Mann $_{M}$ steht an der Ampel. Er ${ }_{M}$ isst ein Eis.

Ein Austauschen des Nomens durch eines mit einem abweichenden Genus erfordert auch die Anpassung der Kongruenzformen. Das Genussystem des Deutschen wird folglich traditionellerweise als Kongruenzsystem beschrieben, in welchem die jeweilige Kongruenzform auf Grundlage des Nomens bestimmt wird und das Genus dem Nomen inhärent ist (vgl. z. B. Ronneberger-Sibold 2004, Hoberg 2004). Diese traditionelle Annahme wurde jedoch in letzter Zeit immer wieder in Frage gestellt und durch eine nicht-lexikalische Verortung des Genus ersetzt (vgl. u. a. Köpcke/Zubin 2009, Nübling 2015). Ein Argument findet sich auch in folgendem Beispiel: Ersetzt man Frau bzw. Mann durch das Neutrum Mädchen, ist die Erzeugung pronominaler Kongruenz auf unterschiedliche Weise möglich (3a bzw. 3b), woraus variierende Kongruenzformen resultieren.

(3a) Ein $_{\mathrm{N}}$ nettes $_{\mathrm{N}}$ Mädchen $\mathrm{N}_{\mathrm{N}}$ steht an der Ampel. Es $\mathrm{s}_{\mathrm{N}}$ isst ein Eis.

(3b) Ein $_{\mathrm{N}}$ nettes $_{\mathrm{N}}$ Mädchen $_{\mathrm{N}}$ steht an der Ampel. Sie F $_{\mathrm{F}}$ isst ein Eis.

Innerhalb der NP wird denjenigen Formen, die Kongruenz erfordern, jeweils der Genuswert N zugewiesen. Außerhalb der NP kann jedoch sowohl ein neutral (3a) als auch ein feminin (3b) kongruierendes Pronomen gewählt werden, um auf Mädchen zu referieren. Diese Möglichkeit ergibt sich aus dem Status von Mädchen als hybrides Nomen: Es liegt eine Genus-Sexus-Divergenz vor, das bedeutet, dass die übliche Merkmalskombination Femininum und [+weiblich] hier nicht gegeben ist (vgl. Corbett 1991).

Die Hybridität wird durch die Kongruenzform abgebildet, denn grammatisch betrachtet bleibt Mädchen ein Neutrum. Erst bei der Pronominalisierung jener Nomina kann das Genus aufgrund der Genus-Sexus-Konkurrenz variieren. Dabei entsteht im Zuweisungssystem ein Konflikt. Die Kongruenz kann aufgrund der unterschiedlichen genusdeterminierenden Merkmale auf zwei Ebenen hergestellt werden. Mädchen als Neutrum ermöglicht einerseits die grammatische Kongruenz, welche infolge des Genuswertes N von Mädchen vergeben werden kann. Darüber hinaus kann Sexuskongruenz aufgrund der konzeptuellen Repräsentation des Referenten [+weiblich = F] hergestellt werden. Für die Beschreibung dieses Phänomens werden unterschiedliche Termini verwendet: Corbett (1991) verwendet für die Beschreibung der Kongruenzformen die Termini syntactic und semantic agreement. Köpcke (2012) 
wählt die Termini grammatische und pragmatische Kongruenz, Oelkers (1996) grammatische und biologische Kongruenz. Für diese Untersuchung wurden die Begriffe Genus- und Sexuskongruenz gewählt. Zur Beschreibung dieser syntagmatischen Beziehungen von Nomen und Pronomen werden von Corbett (2006) die Begriffe Controller (Auslöser) und Target (Ziel) eingeführt. Der Controller kann nicht nur ein Nomen, sondern auch ein Merkmal in der außersprachlichen Welt (z. B. [+weiblich]) sein. Das Target erfordert eine Kongruenzmarkierung. Darüber hinaus liegt die Besonderheit des Targets in der Referenzidentifikation, da diese in der Regel auf den Controller verweisen. Im Fall der sexuskongruenten Targets ergibt sich jedoch eine Abweichung. Es wird auf das außersprachliche Merkmal natürliches Geschlecht des Referenzobjekts verwiesen und nicht auf eine grammatische Eigenschaft des Controllers. Dabei bezeichnen Controller und Target dasselbe außersprachliche Referenzobjekt: Es liegt Koreferenz vor. Durch die Wahl des sexuskongruenten Targets wird allerdings nicht direkt auf den Controller verwiesen, sondern auf dessen außersprachliche Konzeptualisierung. Dieser Konflikt führt bei nicht attributiven Targets zu der Variation von genus- und sexuskongruenten Formen.

Die auf hybride Nomina referierenden Kongruenzformen und die Einflussfaktoren für deren Auswahl wurden bereits in einigen Untersuchungen und aus unterschiedlichen Perspektiven thematisiert. Das Kongruenzverhalten in Abhängigkeit des Targettyps kann mithilfe der Agreement Hierarchy beschrieben werden. Abgebildet wird das Kontinuum zwischen Genus- und Sexuskongruenz in Bezug auf die Wortart des Targets (vgl. Corbett 1991, 2006; Köpcke, Panther \& Zubin 2010). Als Ergebnis seiner Untersuchungen schlägt Corbett (2006) folgende Anordnung der Elemente innerhalb der Hierarchie vor, die universalsprachlich gelten soll:

attributive $>$ predicate $>$ relative pronoun $>$ personal pronoun

Je weiter rechts das Target auf dem Kontinuum angeordnet ist, desto wahrscheinlicher weist es Sexuskongruenz auf. Für die deutsche Sprache spielt lediglich die zweite genannte Kategorie predicate keine Rolle, da die Kategorie Genus in der verbalen Flexion nicht abgebildet wird. Köpcke und Zubin (2009) haben auf dieser Grundlage eine Hierarchie für das Deutsche entwickelt, welche als Endpunkte der Skala die Werte grammatikalisch (Determinierer, Attribut) und konzeptuell (deiktisches Pronomen) aufweist. Die Kongruenzformen außerhalb der NP sind auf syntaktischer Ebene nicht eng an den Controller gebunden und als autonom zu betrachten. Es werden NP-extern daher lediglich die Kategorien Numerus und Genus abgebildet und nicht Kasus. Da die Variation bei NP-externen Kongruenzformen im Deutschen sowohl synchron als auch diachron am stärksten ausgeprägt ist, fokussieren bisherige Studien zu den 
die Variation bedingenden Einflussfaktoren vor allem diesen Bereich. Allerdings ist die Anzahl quantitativer Studien zu diesem Thema überschaubar (vgl. u.a. Birkenes, Chroni \& Fleischer 2014; Czech 2015; Fleischer 2012).

Bisherige Versuche, die Variation von genus- vs. sexuskongruenten Targets zu systematisieren, haben zwei Einflussfaktoren als bedeutsam herausgestellt: Einerseits den bereits durch die Agreement Hierarchy abgebildeten pronominalen Typus des Targets, welcher im Deutschen Relativ-, Demonstrativ-, Possessiv- oder Personalpronomen entspricht (vgl. Köpcke, Panther \& Zubin 2010). Andererseits spielt die lineare Distanz zwischen Controller und Target (also der Abstand in Token oder Silben) eine bedeutende Rolle (vgl. Thurmair 2006; Birkenes, Chroni \& Fleischer 2014; Oelkers 1996).

Birkenes, Chroni \& Fleischer (2014) zeigen in ihrer umfassenden Studie anhand fünf unterschiedlicher Controller für die Zeitspanne vom 17. bis zum 19. Jahrhundert in literarischen Texten den Einfluss des pronominalen Typus und bestätigen damit die Anordnung auf dem Kontinuum der Agreement Hierarchy.

Köpcke \& Zubin (2009), Panther (2009) sowie Thurmair (2006) fokussieren in ihren Untersuchungen den Einfluss der linearen Distanz auf die Kongruenzformen. Je größer die lineare Distanz zwischen Controller und Target ist, desto wahrscheinlicher wird die Wahl eines sexuskongruenten Targets. Köpcke \& Zubin (2009: 142) nennen als möglichen Grund dafür den schwindenden Aktivierungsgrad des grammatischen Controllers im Sprachverarbeitungsprozess.

Über die diachrone Entwicklung des Phänomens existieren in der Forschungsliteratur kontroverse Aussagen. Seit dem Ende des Frühneuhochdeutschen hat sich die Kongruenz der hybriden Nomina laut Birkenes, Chroni \& Fleischer (2014) nicht mehr signifikant verändert. Lediglich die sexuskongruenten Targets in attributiver Position nehmen konstant ab. Auch Dal \& Eroms (2014: 184) vertreten die Ansicht, dass die Sexuskongruenz eher in den älteren Sprachstufen zu verorten sei und daher zunehmend abgebaut werde. Oelkers (1996) hingegen geht von einem zunehmenden Ausbau der Sexuskongruenz aus.

Die genannten Untersuchungen erklären die Wahl der Kongruenzform durch Faktoren wie Pronomentyp und lineare Distanz. Es existieren nur sehr wenige Untersuchungen, die als mögliche Einflussfaktoren solche in Betracht ziehen, die außerhalb der Grammatik liegen, insbesondere quantitativ angelegte Studien sind rar. Braun \& Haig (2010), Panther (2009) und Robinson (2010) ziehen neben den traditionellen grammatischen Einflussfaktoren die Konzeptualisierung des Diskursreferenten als möglichen Einflussfaktor in Betracht. So wird als eine mög- 
liche Erklärung für die Variation der Kongruenzformen auch der Faktor Alter der Referenzfigur genannt. Diese außerhalb der Grammatik liegenden Einflussfaktoren werden in diesem Beitrag in den Blick genommen. In der Einleitung zu diesem Band weisen die Herausgeberinnen bereits auf die zahlreichen Anknüpfungspunkte zur Prototypentheorie in den unterschiedlichsten Bereichen der sprachwissenschaftlichen Forschung hin. Neben den Bereichen Spracherwerb und Sprachwandel wurden Prototypen in erster Linie als Erklärung im Bereich der (Flexions)Morphologie angewendet. (vgl. Einleitung i.d. B.: 3) In dem vorliegenden Beitrag wird eine Prototypizitätsskala als Erklärung für die variierenden Kongruenzformen hybrider Nomina genutzt. Ausgehend von der Agreement Hierarchy (vgl. Corbett 1991, 2006; Köpcke, Panther \& Zubin 2010) wird von einem prototypischen Kontext für sexuskongruente Targets ausgegangen, welcher sowohl grammatische als auch außergrammatische Merkmale umfasst.

\section{Fragestellung und Annahmen}

Im Folgenden soll untersucht werden, welche nicht-grammatischen Faktoren Einfluss auf die Kongruenzformen nehmen. Dabei wird angenommen, dass die Sexualisierung innerhalb des Kontexts eine bedeutende Rolle spielt. Ausgangspunkt für die im weiteren Verlauf näher erläuterten Annahmen sind die beiden folgenden Sätze aus Die Zeit aus den Jahren 2011 und 2012.

(4) Immerhin ist das dänische Model $_{N}$ bekannt für seinen ${ }_{N}$ androgynen Stil.

(Die Zeit, 09.03.2011)

(5) Und weil so ein Model $_{N}$ eben zeigt, was sie $\boldsymbol{F}_{\mathrm{F}}$ zeigen kann, hat sie $_{\mathrm{F}}$ auf dem Foto nicht sehr viel an.

(Die Zeit, 27.02.2012)

In beiden Sätzen liegt als grammatischer Controller das Neutrum Model vor, welchem als Referenzperson in der außersprachlichen Welt jeweils das Merkmal [+weiblich] zugeschrieben werden kann. ${ }^{1}$ Auf Model kann folglich mithilfe von genus- oder sexuskongruenten Pronomina verwiesen werden. Bei der Analyse der Beispielsätze können zunächst die traditionellen, grammatischen Einflussfaktoren

1 Model kann natürlich sowohl auf Männer als auch auf Frauen referieren. Im weiteren Kontext dieser Zeitungsartikel wird jedoch deutlich, dass mit Model hier jeweils eine Frau bezeichnet wird. 
angeführt werden. Dabei wird deutlich, dass sich bei diesen Beispielen weder das Erscheinungsjahr (2011 bzw. 2012) noch die Distanz (zwei bzw. drei Tokens) zwischen Target und Controller maßgeblich unterscheiden. Lediglich der pronominale Typus variiert, da in (4) ein Possessivpronomen und in (5) ein Personalpronomen vorliegt. Hinsichtlich ihres Kongruenzverhaltens sind diese beiden pronominalen Targets laut Agreement Hierarchy jedoch vergleichbar. Für die Erklärung dieses Phänomens kann nur bedingt auf grammatische Einflussfaktoren zurückgegriffen werden. Eine mögliche Erklärung könnten unter Umständen die unterschiedlichen syntaktischen Domänen der Targets sein. Bei Betrachtung des Kontextes der Kongruenzformen fällt aber auf, dass die Kongruenzformen auch durch ebenjenen motiviert sein könnten. In (4) wird die Androgynität des weiblichen Models beschrieben. Im Gegensatz dazu ist das Model in Beispiel (5) sowie in der den Text begleitenden Abbildung fast nackt.

Anhand einer Korpusuntersuchung soll im Folgenden gezeigt werden, dass diese Beobachtung kein Einzelfall ist und dass die Wahl des Kongruenzwertes nicht ausschließlich mithilfe der Einflussfaktoren lineare Distanz und pronominaler Typus zu erklären ist, sondern, dass auch der Kontext eine Rolle spielt. Das Genus des Targets wird demnach auch aufgrund der semantischen Verortung des Controllers vergeben. Es soll überprüft werden, ob ein sexueller beziehungsweise erotischer pragmatischer Kontext die Verwendung sexuskongruenter Formen befördert.

\section{Daten}

Die aufgestellte Hypothese soll anhand des hybriden Nomens Mädchen überprüft werden. ${ }^{2}$ Die Entscheidung für Mädchen fiel in erster Linie aufgrund der neutralen Denotation, die nichtsdestotrotz unterschiedliche Konnotationen zulässt. So kann Mädchen sowohl auf eine geschlechtsunreife weibliche Person als auch auf geschlechtsreife weibliche Personen referieren und sowohl positiv als auch negativ

\footnotetext{
2 Es existieren natürlich noch weitere Personenbezeichnungen mit Genus-Sexus-Divergenz. Das gilt sowohl für weibliche Referenzpersonen wie beispielsweise Mädchen $_{\mathrm{N}}$ oder $\operatorname{Vamp}_{\mathrm{M}}$ als auch für männliche wie Jungchen ${ }_{\mathrm{N}}$ oder Schwuchtel $_{\mathrm{F}}$ (vgl. Köpcke \& Zubin 2005). Darüber hinaus existieren Nomina, die nicht sexusspezifisch verwendet werden (z. B. Model), aber nichtsdestotrotz eine Genus-Sexus-Divergenz aufweisen können. Jedoch werden diese aufgrund ihrer Sexusindifferenz nicht immer zu den hybriden Nomina gezählt.
} 
verwendet werden. Der Duden ${ }^{3}$ nennt in der Bedeutungsübersicht als mögliche Bedeutungen für Mädchen sowohl „Kind weiblichen Geschlechts“ als auch „junge, jüngere weibliche Person“ sowie „Freundin (eines jungen Mannes)“. Darüber hinaus handelt es sich bei Mädchen um ein hybrides Nomen mit vergleichsweise hoher Frequenz. Bei anderen ebenfalls vergleichsweise frequenten hybriden Nomina wie Weib oder Fräulein ist der Bedeutungsspielraum sehr viel eingeschränkter.

Um die Prozesse aufzudecken, die der Auswahl der Kongruenzformen hybrider Nomina zugrunde liegen, muss eine geeignete Datengrundlage ausgewählt werden. Für diese Untersuchung fiel die Wahl auf Texte aus unterschiedlichen Korpora geschriebener Sprache. Dabei ist es von Bedeutung, dass Texte des gleichen Texttyps, jedoch von unterschiedlichen Autoren zugrunde gelegt werden, um eventuelle individuelle Entscheidungen einzelner Autoren auszuschließen.

Das ausgewählte Korpus setzt sich aus zwei Subkorpora zusammen: literarische Texte des Neuhochdeutschen (Subkorpus A) und journalistische Texte (Subkorpus B).

Ausgewählt wurde zunächst ein Korpus mit literarischen Texten. Dabei wurde in Anlehnung an die von Birkenes, Chroni \& Fleischer (2014) durchgeführte Studie zu der Entwicklung der Kongruenzformen hybrider Nomina auf die digitale Bibliothek „Deutsche Literatur von Luther bis Tucholsky“ zurückgegriffen. ${ }^{4}$ Dieses literarische Korpus des 17. bis 19. Jahrhunderts (Subkorpus A, $N=1600$ Kongruenzformen) besteht aus Texten neun verschiedener Autoren: Abraham a Sancta Clara, Theodor Fontane, Johann Wolfgang von Goethe, Georg Philipp Harsdörffer, E.T.A. Hoffmann, Sophie von La Roche, Daniel Casper Lohenstein, Johann Karl August Musäus und Johann Gottfried Schnabel.

Bei der Erweiterung des Korpus fiel die Entscheidung für das Subkorpus B ( $\mathrm{N}=409$ Kongruenzformen) auf journalistische Texte, da diese deutlich mehr Variation der Kongruenzformen aufweisen. Ausgewählt wurden Texte aus Die Zeit (DeReKo, Zugriff via Cosmas) $)^{5}$ im Zeitraum von 1950 bis 2000. Die Untersuchung ausschließlich literarischer Texte erlaubt zwar interessante

3 Dudenredaktion (o. J.): „Mädchen“ auf Duden online. URL: https://www.duden.de/rechtschrei bung/Maedchen (3. August 2018).

4 Über die Plattform des Texgrid-Korpus (https://textgrid.de/digitale-bibliothek) kann auf diese Daten zugegriffen werden.

5 Das Deutsche Referenzkorpus DeReKo, http://www.ids-mannheim.de/kl/projekte/korpora/, am Institut für Deutsche Sprache, Mannheim. 
Schlussfolgerungen über das Kongruenzverhalten hybrider Nomina, bietet jedoch für eine umfassende Analyse der Einflussfaktoren eine zu geringe Variation der Kongruenzformen.

\section{Annotation}

Da die Kategorie Genus im Deutschen nur im Singular abgebildet wird, kommen als mögliche Controller alle Vorkommen von Mädchen - sowohl als Simplex als auch als Kopf eines Kompositums - im Singular in Frage. Als mögliche Referenzausdrücke annotiert werden Targets in einem Kontext von fünf Zeilen vor und fünf Zeilen nach dem Controller, welche sich außerhalb der NP befinden. Die Einschränkung auf Targets außerhalb der NP begründet sich durch Resultate anderer Untersuchungen (vgl. z. B. Birkenes, Chroni \& Fleischer 2014) und einer eigenen Pilotierung, die zeigen, dass Sexuskongruenz ein Phänomen ist, welches in der Regel bei nicht-attributiven Targets auftritt (s. auch Abschnitt 2). Folglich können Personal-, Demonstrativ-, Possessiv-, RelativInterrogativ- und Indefinitpronomina sowie auch Ordinalzahlen mögliche Kongruenzziele darstellen. Der Kontext vor dem Controller wird miteinbezogen, da in seltenen Fällen Pronomina in kataphorischer Verwendung auftauchen und zudem hierdurch eine bessere Kontextualisierung von Controller und Target möglich ist.

Annotiert wurde die grammatische Struktur mit den Faktoren lineare Distanz zwischen Controller und Target (Anzahl der Tokens) sowie Wortart des Targets. Eine erste Pilotierung der Daten ließ darüber hinaus vermuten, dass der grammatische Faktor Definitheit Einfluss auf die Kongruenzform der Targets nimmt - also ob ein definiter oder indefiniter Controller zugrunde liegt. Aus diesem Grund wurde dieser Faktor hinzugefügt. Ausgewertet wurden nur Targets mit einer eindeutigen Referenz. Die ambigen Fälle wurden als solche markiert und bei der Auswertung nicht weiter berücksichtigt. Das in Beispiel (6) auf Mädchen referierende Personalpronomen ihrem wurde als ambig gekennzeichnet, weil sowohl Mädchen ${ }_{\mathrm{N}}$ als auch Natalie $_{\mathrm{F}}$ als Controller fungieren können. Dies traf für 86 Pronomina des Korpus zu.

(6) Der große Schlag in Richard Bauschs Roman kommt in dem Augenblick, in dem das schöne deutsche Mädchen ${ }_{\mathrm{N}}$ Natalie $_{\mathrm{F}}$ ihrem $_{\mathrm{F}}$ „Verlobten“ Walter sagt [. . .]

(Die Zeit, 05.03.1998) 
Darüber hinaus wurden Faktoren für die Annotation von Sexualität/Erotik benötigt. Da es hierfür keine Standards gibt, wurden diese sukzessive anhand des Materials entwickelt. Um den Faktor der Sexualität operationalisierbar zu machen, wurden mithilfe des von Zinsmeister und Lemnitzer $\left(2015^{3}: 103\right)$ vorgeschlagenen iterativen Annotationszyklus unterschiedliche Indikatoren zur Operationalisierung von Sexualität ermittelt. In einem ersten Schritt erfolgt die Exploration der Daten. Es folgt ein Wechsel von Annotation mit anschließender Analyse, um das Annotationsschema zu evaluieren und anzupassen. Die anschließende Auswertung der Daten erfolgt textorientiert. Mithilfe des Annotationszyklus wurden sukzessive vier Faktoren entwickelt, die versuchen, den Sexualisierungsgrad des Kontexts zu fassen: semantische Rolle, Alter der Referenzfigur, Antagonist und sexuelle Aufladung des Kontexts. Der Faktor semantische Rolle beschreibt mithilfe der Ausprägungen Agens, Patiens, Experiencer, Thema, Locus, Instrument, Stimulus und Rezipient die semantische Rolle des Targets. Es wird angenommen, dass auch das Alter der Referenzfigur einen Einfluss auf die Kongruenzform hat (vgl. Braun \& Haig 2010; Robinson 2010). Aus diesem Grund wurde als weiterer Faktor Alter der Referenzfigur gewählt. Das Alter der Referenzfigur wird oftmals nicht explizit genannt und muss daher aufgrund unterschiedlicher Indikatoren ermittelt werden. Mit dem Wert reif ist in diesem Kontext Geschlechtsreife gemeint. Da das Alter der Referenzfigur in der Regel ein im Abschnitt invariabler Faktor ist, können diese Indikatoren auch Attribute sein, die dem Controller zugeschrieben werden. Darüber hinaus kann das Alter auch aufgrund von Referenzidentitäten rekonstruiert werden. Solange es keine abweichenden Informationen gibt, wird in einem Kontext folglich immer das gleiche Merkmal für das Alter einer Referenzfigur gewählt. Wenn das Alter der Referenzperson anhand des vorliegenden Auszuges nicht eindeutig festgestellt werden kann, wird der Wert mehrdeutig vergeben.

Das deutsche Genussystem kann in Bezug auf Personenbezeichnungen als sexusbasiert beschrieben werden. Um dem Zusammenhang von Genus und Sexus nachzugehen, wurde der Faktor Antagonist mit den Merkmalen männlich, weiblich, nicht vorhanden annotiert, die nach Interaktionspartnern innerhalb des annotierten Kontexts fragt. Schließlich wurde der gesamte Kontext bewertet und einer der drei Werte neutral, Liebe/Erotik, sexuell/pornographisch vergeben. Ein Kontext mit dem Wert Liebe/Erotik zeichnet sich dadurch aus, dass eine romantische oder erotische Handlung stattfindet. Die als sexuell/pornographisch annotierten Kontexte sind dadurch gekennzeichnet, dass eine explizit sexuelle Handlung zwischen dem Mädchen und einem Interaktionspartner stattfindet. Die neutralen Kontexte sind der Default für alle Kontexte, die nicht 
durch die anderen beiden Faktoren abgedeckt sind. Daraus ergibt sich folgendes Annotationsschema (Abb. 1):

\begin{tabular}{ll}
\hline Faktor & Merkmale \\
\hline $\begin{array}{l}\text { lineare Distanz zwischen } \\
\text { Controller und Target in } \\
\text { Anzahl der Tokens }\end{array}$ & $0,1,2,3,4,5,>5,>10,>20$ \\
\hline PoS & PDS, PPER, PPOSAT, PPOSS, PRELAT, PRELS ${ }^{\circ}$ \\
\hline Kongruenz & genuskongruent, sexuskongruent, ambig \\
\hline Referenz & mehrdeutig \\
\hline Antagonist & männlich, weiblich, nicht vorhanden \\
\hline semantische Rolle & $\begin{array}{l}\text { Agens, Patiens, Experiencer, Thema, Locus, Instrument, Stimulus, } \\
\text { Rezipient }\end{array}$ \\
\hline Alter der Referenzfigur & kindlich, pubertär, reif, ambig \\
\hline Definitheit & definit, indefinit \\
\hline $\begin{array}{l}\text { Sexualisierung } \\
\text { des Kontexts }\end{array}$ & neutral, Liebe/Erotik, sexuell/pornographisch \\
\hline
\end{tabular}

Abb. 1: Annotationsschema.

Da die Operationalisierung von Sexualität auf einigen interpretativen Entscheidungen beruht, wird die Stringenz der Annotation durch „intrarater reliability“ (vgl. Diekmann 2009) abgesichert. Die erste Annotation wurde ergänzt durch eine weitere Stichprobenannotation nach drei Monaten. Da diese lediglich eine Abweichung von $3 \%$ ergeben hat, wurde die erste Annotation als reliabel eingeschätzt.

\section{Literarische Texte (Subkorpus A)}

Eine erste Annotation und die folgende Auswertung der Daten aus dem Subkorpus A haben ergeben, dass die deutlichste Variation zwischen den Domänen NP-intern und NP-extern festzustellen ist. In attributiver Position werden ausschließlich neutrale, also genuskongruente, Targets gewählt, in nicht-attributiver Position gibt es schließlich Variation zwischen genus- und sexuskongruenten Targets. Demnach muss grundsätzlich bei allen Aussagen zum Kongruenzverhalten zwischen attributiver und anaphorischer Kongruenz

6 PDS = substituierendes Demonstrativpronomen; PPER = Personalpronomen; PPOSAT = attribuierendes Possessivpronomen (mit Mädchen als Possessor); PREL = Relativpronomen. 
unterschieden werden. Diese Ergebnisse haben auch dazu geführt bei den folgenden Analysen die attributiven Elemente unberücksichtigt $\mathrm{zu}$ lassen und lediglich die pronominalen Kongruenzformen $\mathrm{zu}$ fokussieren.

Es wird deutlich, dass - unabhängig vom pronominalen Typus - der Anteil der sexuskongruenten Targets sehr hoch ist (92\%) und dass damit außerhalb der NP nur geringe Variation zwischen genus- und sexuskongruenten Targets vorliegt. Lediglich bei den Relativpronomina (PREL) lässt sich erhebliche Variation im Kongruenzverhalten beobachten. Bemerkenswert ist an dieser Stelle, dass trotz der in der Regel unmittelbaren Nähe zum Controller $42 \%$ der Relativpronomina Sexuskongruenz aufweisen. Die Personalpronomina (PPER) und Possessivpronomina (PPOSAT) verhalten sich zu jeweils $97 \%$ sexuskongruent. Aufgrund des hohen Anteils der sexuskongruenten Targets wurde das Korpus erweitert, um somit die Anzahl der zwischen Genus- und Sexuskongruenz variierenden Targets zu erhöhen.

\section{Journalistische Texte (Subkorpus B)}

Bei der Gesamtauswertung der Daten fällt zunächst auf, dass die beiden Subkorpora A und B sich im Hinblick auf das Kongruenzverhalten stark unterscheiden. Während in den literarischen Texten des 17. bis 19. Jahrhunderts die Personalpronomina (PPER) zu 97\% sexuskongruente Targets aufweisen (Abb. 2), liegt bei den PPER in den journalistischen Texten mit $70 \%$ sexuskongruenten Targets stärkere Variation vor. Eine Vergleichbarkeit der Korpora in Bezug auf ihre Kongruenzformen ist jedoch aufgrund der unterschiedlichen Jahrhunderte und der unterschied-

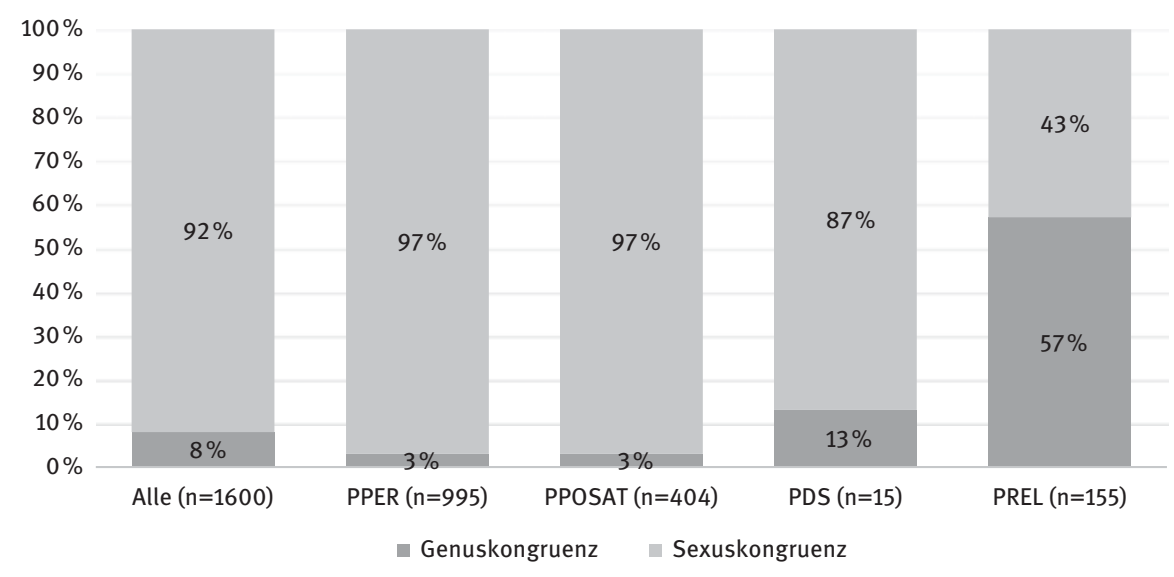

Abb. 2: Kongruenzformen in Abhängigkeit des Pronomens, literarische Texte. 
lichen Textsorten nicht gegeben. Es kann nur vermutet werden, dass eine mögliche Erklärung für die $30 \%$ genuskongruenten Targets einerseits die größere Normorientierung in den journalistischen Texten sein kann. Beim Blick in den Duden (vgl. Gallmann 2009) finden sich nur zwei explizite Regeln für die Kongruenz bei hybriden Nomina: Die Relativpronomina übernehmen das Genus des Bezugswortes und bei den Personalpronomina kann mit dem natürlichen Geschlecht referiert werden, allerdings nur bei höherer Distanz zwischen Target und Controller.

Andererseits ist auch die bewusste Nutzung von sexuskongruenten Formen sowohl in den literarischen Texten als auch in den journalistischen Texten durch die Autoren denkbar. Für die Autoren literarischer Texte scheinen diese Normen unter bestimmten Umständen nur eine untergeordnete Rolle zu spielen. Unabhängig vom pronominalen Typus und der Distanz zwischen Controller und Target sowie möglichen pragmatischen Einflussfaktoren scheint die Wahl der Kongruenzformen auch von der Textsorte abhängig zu sein. Darüber hinaus könnten auch die Realisierungsformen der Sprache Einfluss auf die Kongruenzformen nehmen. Mit der Einschränkung des Korpus auf geschriebene Sprache können folglich nur Aussagen zum Kongruenzverhalten in der geschriebenen Sprache (journalistische Texte) getroffen werden.

\subsection{Grammatische Einflussfaktoren}

Die Auswertung der Targets nach pronominalem Typus $(\mathrm{N}=487)(\mathrm{Abb} .3)$ bestätigt die gemeinhin angenommene Agreement Hierarchy (vgl. Corbett 1991, 2006;

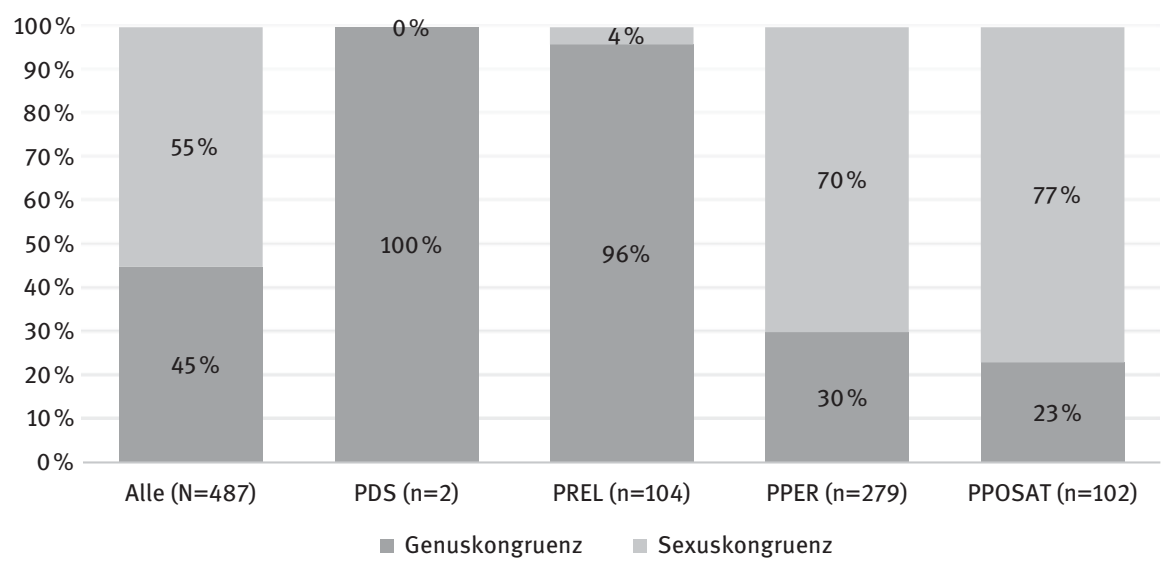

Abb. 3: Kongruenzformen in Abhängigkeit vom pronominalen Typus, journalistische Texte. 
Köpcke, Panther \& Zubin 2010). Die Targets lassen sich in Abhängigkeit von ihrem pronominalen Typus auf einem Kontinuum abbilden, in welchem die Tendenz zur Sexuskongruenz von den Demonstrativ- und Relativpronomina hin zu den Personal- und Possessivpronomina deutlich zunimmt.

Die Aufschlüsselung des Kongruenzverhaltens in Abhängigkeit der linearen Distanz zwischen Controller und Target (Abb. 4) zeigt, dass mit zunehmendem Abstand auch die Wahrscheinlichkeit zunimmt, dass ein sexuskongruentes Target verwendet wird.

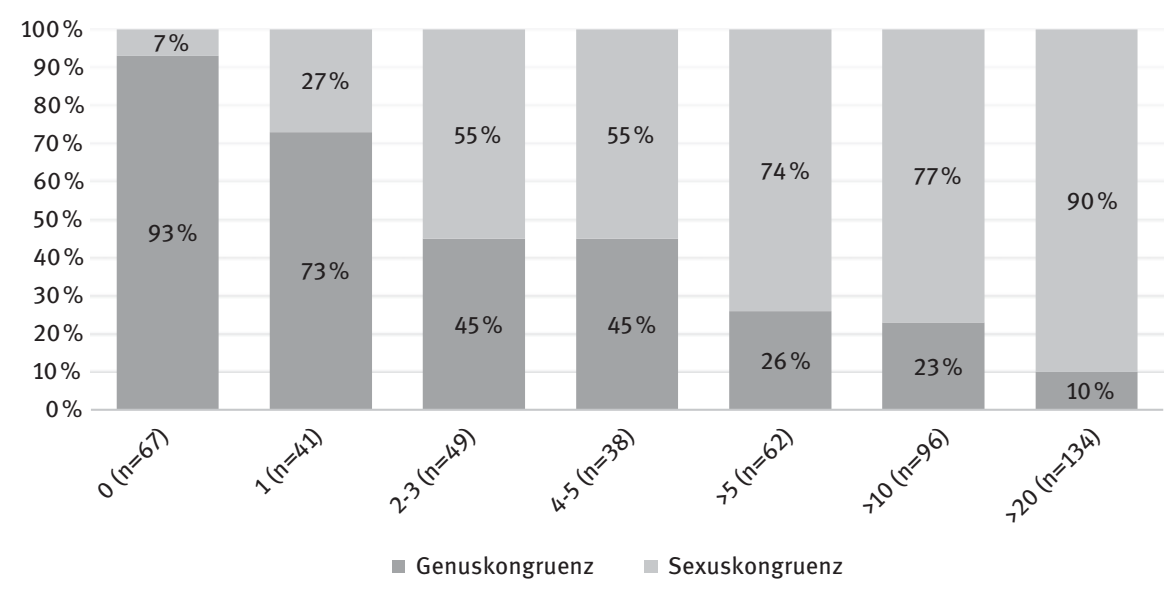

Abb. 4: Kongruenzformen in Abhängigkeit von der linearen Distanz, journalistische Texte.

Der Einfluss der linearen Distanz zeigt sich auch in Referenzketten (Bsp. 7), die zunächst mit einem genuskongruenten Target beginnen und dann mit zunehmender Distanz einen Wechsel zu einem sexuskongruenten Target aufweisen.

(7) Ein Mädchen ${ }_{N}$ stand auf den Trümmern, wir sahen es $s_{N}$ schon von weitem, ohne Mantel, mit rotgefrorenem Gesicht. Mit bloßen Händen grub sie $e_{\mathrm{F}}$ planlos im Schnee.

(Die Zeit, 21.01.1954)

\subsection{Pragmatische Einflussfaktoren}

Bei der Auswertung der Faktoren wurden aufgrund der geringen Belegzahlen $(\mathrm{N}=487)$ alle Targets zusammengefasst. Die Auswertung der einzelnen Faktoren zur Operationalisierung der Sexualisierung des Kontextes hat ergeben, dass der Faktor semantische Rolle in dem gewählten Korpus keinen Einfluss auf die Form 
der Targets hat. Der Einfluss des Kontexts kann stark variieren: Er kann als zusätzlicher Faktor zu den grammatischen bis hin zum alleinigen steuernden Element für die Kongruenzform wirksam werden. Entscheidend für die Operationalisierung des Faktors Sexualität ist die Kombination der anderen drei analysierten Faktoren Alter der Referenzfigur, Antagonist und Sexualität (Abb. 5 und Abb. 6). Die Einzelauswertung dieser Faktoren zeigt, dass nicht alle für sich genommen Einfluss auf die Wahl der Kongruenzform haben (Abb. 5), sondern dass Kontexte existieren, in denen nur einige der Parameter auf Sexualisierung hinweisen. Eine Ausnahme dazu stellt der Faktor Alter der Referenzfigur dar.

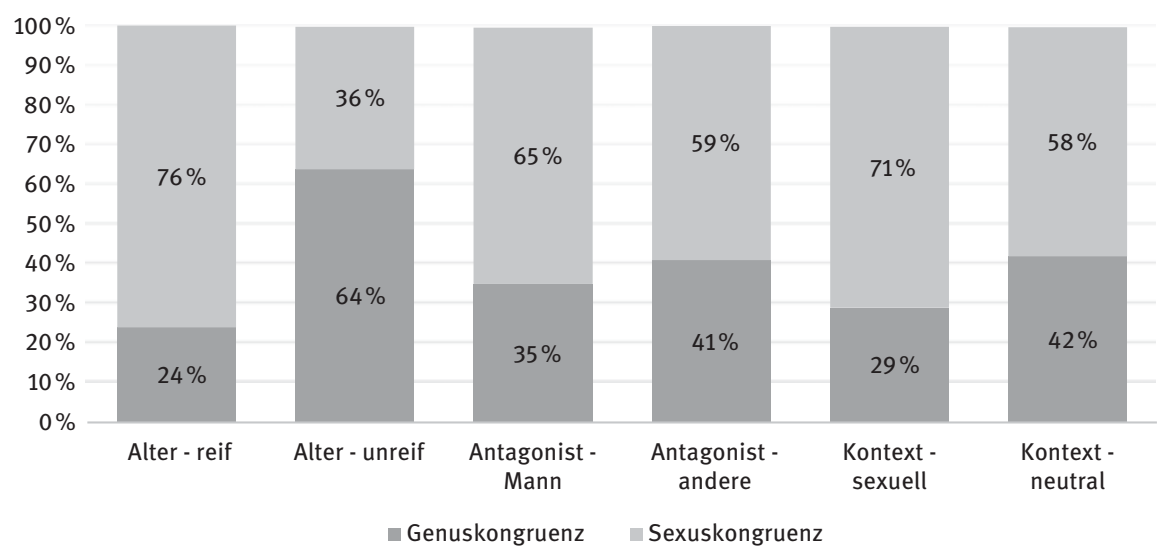

Abb. 5: Kongruenzformen in Abhängigkeit der pragmatischen Faktoren.

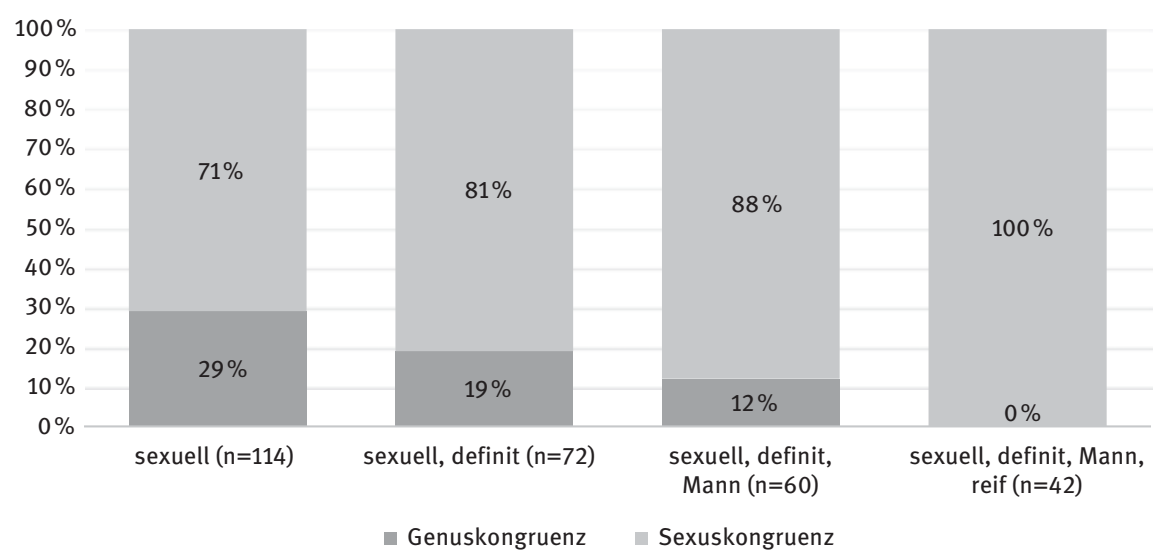

Abb. 6: Kongruenzformen in Abhängigkeit des Kontexts. 
Wenn es sich um ein sexuell reifes Mädchen handelt, weisen 76,3\% der Targets Sexuskongruenz auf, bei den als kindlichen klassifizierten Verwendungen nur 35,6\%. Diese Ergebnisse fügen sich in das Bild, welches Busley \& Fritzinger (2018) in ihrer Studie zur Korrelation von Geschlechtsreife und Genus der Femineutra (Frauen in Verbindung mit dem Neutrum) beschreiben. Für die Faktoren Antagonist und Kontext liegt der Anteil der genus- bzw. sexuskongruenten Targets sehr nah beieinander.

Bei der Annotation des Kontexts handelt es sich nicht um eine binäre Opposition. Aus diesem Grund wird ein Kontinuum angenommen. Die Faktoren wirken lediglich in Kombination und bilden eine Art Kontinuum der Sexualisierung des Textes - zwischen den Polen neutral und explizite Sexualität - auf welchem das Target innerhalb seines Kontexts eingeordnet werden kann. Je mehr Faktoren den Kontext als sexuell kennzeichnen, desto wahrscheinlicher ist die Realisierung eines sexuskongruenten Pronomens (Abb. 6). Im Folgenden wird der Einfluss des Kontexts an der Gesamtheit aller Targets $(\mathrm{N}=487)$ illustriert, da eine Aufteilung der Targets nach linearer Distanz und Targettyp ein zu geringes n zur Folge gehabt hätte. Wenn lediglich der allgemeine Kontext des Pronomens als sexuell/pornographisch annotiert wurde, wird in $71 \%$ der Fälle ein sexuskongruentes Pronomen gewählt. Je mehr Faktoren kombiniert auftreten, desto größer wird die Wahrscheinlichkeit für ein sexuskongruentes Target: Wenn der Kontext als sexuell/pornographisch annotiert wurde, das Pronomen auf einen definiten Controller verweist, ein männlicher Antagonist vorhanden ist und Mädchen zudem als reif beschrieben wird, wird zu 100 \% ein sexuskongruentes Pronomen verwendet. Der Faktor Definitheit trägt zwar nicht zur Sexualisierung des Kontexts bei, nichtsdestotrotz erhöht er die Wahrscheinlichkeit eines sexuskongruenten Targets und wird deshalb an dieser Stelle mit aufgeführt. Wird dieser Faktor unberücksichtigt gelassen, verändern sich die Ergebnisse lediglich um wenige Prozentpunkte, die Tendenz ist jedoch gleichbleibend: Auch ohne den definiten Controller liegen bei der Kombination aller Faktoren 100 \% sexuskongruente Targets vor.

So wird beispielsweise in Beleg (8) trotz der geringen linearen Distanz mithilfe zweier sexuskongruenter Targets (sie, ihr) auf Mädchen verwiesen. Die Wahl der sexuskongruenten Targets lässt sich durch einen Blick auf die nichtgrammatischen Faktoren erklären. Die beschriebene Situation ist für beide Targets als sexuell zu werten, es wird auf ein definites Nomen verwiesen, mit dem Diener ist jeweils ein männlicher Gegenspieler vorhanden und Mädchen referiert auf Katharina, eine Frau in heiratsfähigem Alter.

Im Kontrast dazu steht Beleg (9), in welchem der Kontext als neutral annotiert wurde, die Pronomina auf ein indefinites, unreifes Mädchen verweisen und es keinen männlichen Gegenspieler innerhalb des Kontexts gibt. In diesem 
Beispiel wird auch mit zunehmender linearer Distanz konstant mithilfe von genuskongruenten Pronomina auf Mädchen verwiesen.

(8) Da macht sich Petruchios schrathafter, ekliger Diener (Heinz Schubert) geil an das Mädchen $_{N}$ heran, befingert sie ${ }_{F}$, kriecht ihr ${ }_{F}$ unter den Rock.

(Die Zeit, 11.09.1981)

(9) Dann gibt es da noch ein Mädchen ${ }_{N}$ von zehn Jahren, das ${ }_{N}$ seine $_{N}$ einstudierte Rolle sehr erfolgreich spielt. Es $s_{N}$ steht in Hauseingängen, so morgens zwischen sieben und neun.

(Die Zeit, 02.01.1959)

Eine abschließende Korrelation der Faktoren lineare Distanz in Token und Kontext (Abb. 7) zeigt, dass der schon bekannte Einflussfaktor Distanz zwar Einfluss auf die Kongruenzform nimmt, der Faktor Sexualität jedoch ebenso eine bedeutende Rolle spielt. Um eine größere Menge an Targets zu erhalten, wurden die Targets in einer Distanz von zwei bis drei und vier bis fünf Tokens zu einer Gruppe und die Targets mit einer Distanz von mehr als fünf Tokens zu einer weiteren Gruppe zusammengefasst. Dieses Vorgehen ist m. E. aufgrund der Analyse der Distanz in Tokens (Abb. 4) legitim, da diese gezeigt hat, dass diese pronominalen Target-Typen ein sehr ähnliches Kongruenzverhalten aufweisen. In Bezug auf die möglichen PoS der Targets stehen in dieser Distanz vorzugsweise Personalpronomina und Possessivpronomina. Auch hier zeigt sich zwar, dass mit zunehmender linearer Distanz zwischen Controller und Target die Wahrscheinlichkeit für ein sexuskongruentes Pronomen steigt. Jedoch erhöht die Einbeziehung des Faktors sexualisierter Kontext - insbesondere bei den

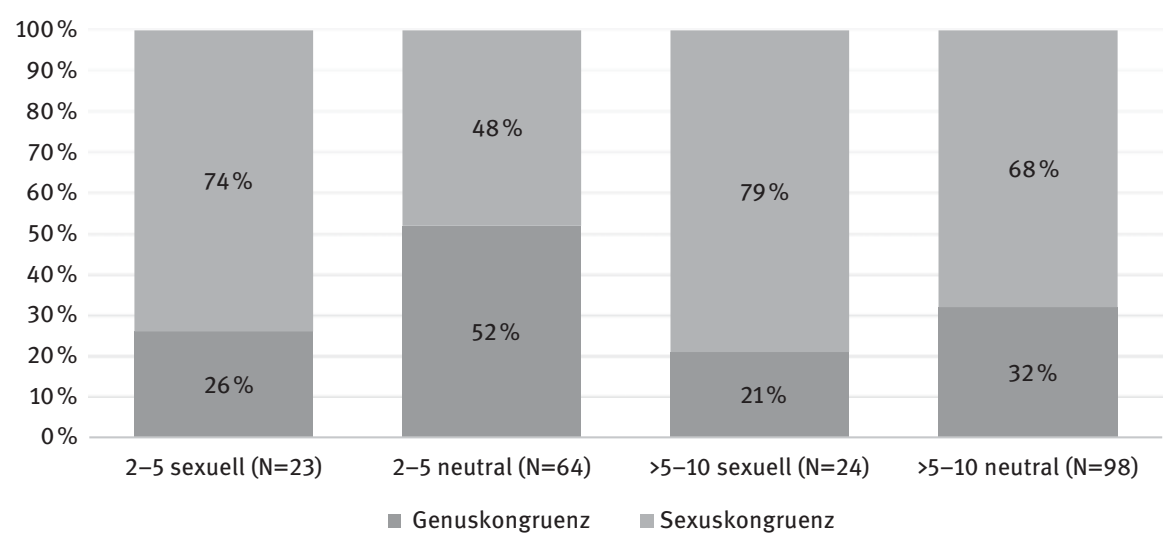

Abb. 7: Korrelation der Faktoren Distanz und Kontext. 
Targets in vergleichsweise geringer Distanz - den Anteil der sexuskongruenten Targets. Bei gleicher Distanz liegen mehr sexuskongruente Pronomina vor, wenn der Kontext als sexuell markiert wurde.

Die Variation der Kongruenzformen lässt sich schließlich am umfassendsten durch die Interaktion von grammatischen und pragmatischen Faktoren erklären. Je nach Kombination dieser Faktoren wird eine bestimmte Kongruenzform in höherem oder geringerem Maße ausgelöst. In manchen Kontexten sind sowohl genus- als auch sexuskongruente Pronomina akzeptabel. Dem gegenüber stehen andere Kontexte, in denen Sprecherinnen und Sprecher durch den Kontext zu einem sexuskongruenten Target geradezu gezwungen werden. ${ }^{7}$ Das führt zu unterschiedlichen Kongruenzformen für ähnliche sprachstrukturelle Gegebenheiten.

Um eine passende Kongruenzform zu wählen, können Sprecherinnen und Sprecher neben den grammatischen Informationen über Distanz und Wortart des Targets auch auf eine pragmatische Projektion zurückgreifen, welche durch den geäußerten Kontext entstanden ist. Diese Projektion wird nicht nur durch offensichtliche Faktoren wie Geschlecht und Alter der Referentin oder des Referenten gespeist, sondern auch durch die restlichen kontextuellen Bedingungen. Es können für jeden der Faktoren Distanz, pronominaler Typus und pragmatischer Kontext Einzelkontinua mit den Polen Genus- und Sexuskongruenz angenommen werden. Die jeweiligen Kontexte können auf diese Weise auf einer Prototypizitätsskala angeordnet werden. Grundlage dieser Prototypizitätsskala bildet die Agreement Hierarchy (vgl. Corbett 1991, 2006; Köpcke, Panther \& Zubin 2010). Diese wird jedoch um die Faktoren lineare Distanz und pragmatischer Kontext ergänzt. Je mehr der Einzelfaktoren auf dem Kontinuum in Richtung Sexuskongruenz angeordnet werden können, desto wahrscheinlicher ist die Wahl eines sexuskongruenten Targets. Die prototypischen Merkmale für Sexuskongruenz sind:

- Possessiv- oder Personalpronomen

- große Distanz zum Controller

- Sexualisierung des Kontexts

- reife Referenzfigur

- männlicher Antagonist

- Verweis auf ein Definitum

7 Ob die Faktoren die Wahl der Kongruenzform unbewusst beeinflussen oder ob die Schreiberinnen und Schreiber sich bewusst für eine bestimmte Kongruenzform entscheiden, kann in diesem Rahmen nicht geklärt werden. 
Der prototypische Kontext für sexuskongruente Targets umfasst folglich sowohl grammatische als auch pragmatische Merkmale. Je mehr Eigenschaften des Prototyps erfüllt sind, desto wahrscheinlicher ist eine zutreffende Einschätzung des Kongruenzverhaltens. Darüber hinaus kann mithilfe des Prototyps auch erklärt werden, warum durchaus ein prototypisches Merkmal für eine sexuskongruente Form bei einem grammatisch kongruierenden Target vorkommen kann. Kein Merkmal bewirkt alleine genommen auf jeden Fall Sexuskongruenz. Es handelt sich um keine kategorielle Einordnung, nach welcher ein sexueller Kontext oder große Distanz zwischen Controller und Target Sexuskongurenz auslösen muss (vgl. Rosch 1972, 1975). Es erfolgt ein Abgleich mit den Eigenschaften des Prototyps: Je mehr Übereinstimmung mit den Merkmalen des Prototyps vorliegt, desto größer ist die Wahrscheinlichkeit für ein sexuskongruentes Target.

Die Daten zeigen darüber hinaus, dass ein Abweichen von der Genuskongruenz auch zur eindeutigen Unterscheidung von Referenzpersonen dienen kann: Bei einem Synkretismus von neutralem und maskulinem Pronomen wird lediglich in $25 \%$ der Fälle das zum Maskulinum homonyme neutrale Pronomen gewählt, in allen anderen Fällen wird die Homonymie umgangen und ein sexuskongruentes, feminines Pronomen gewählt. Trotz der geringen Distanz von nur einem Token zwischen Controller und Target wird in Beispiel (10) mit einem sexuskongruenten Pronomen auf Mädchen verwiesen. In Beispiel (11) liegt zwar eine große lineare Distanz zwischen Controller und Target vor, aber das Mädchen wird explizit als kindlich beschrieben - gleichwohl wird statt des homonymen genuskongruenten Pronomens das sexuskongruente gewählt.

(10) Ein geniales Mädchen $_{\mathrm{N}}$, von ihren F $_{\mathrm{F}}$ übermächtigen Brüdern geliebt, bewundert, behütet und zärtlichst bevormundet, bis sie $e_{\mathrm{F}}$ unter all den erdrückenden Zuwendungen selber keine Luft mehr bekam.

(Die Zeit, 27.09.1991)

(11) Das etwa vierjährige Mädchen ${ }_{\mathrm{N}}$ griff sich einen Ball, drehte ihn, lockerte die Hüfte, warf mit Schwung das Bein nach hinten und beförderte schließlich das harte Leder in Richtung ihrer ${ }_{\mathrm{F}}$ jüngeren Brüder. （Die Zeit, 25.05.1990)

\section{Fazit}

Die Ergebnisse zum Kongruenzverhalten der auf hybride Nomina referierenden Targets ermöglichen einen Einblick in die mentale Organisation des Genussystems des Deutschen. Eine für die Theoriebildung wichtige Frage ist, woher 
Sprecherinnen und Sprecher bei der Formung einer NP die Genusmerkmale beziehen. Traditionell wird angenommen, dass das Genus im Lexikon als Information zusammen mit dem Nomen gespeichert ist (vgl. Brinkmann 1962; Eisenberg 2013; Hoberg 2004). Bei dieser lexikalischen Verortung des Genus ist das Genus eine inhärente und demnach auch invariante Eigenschaft des Nomens, welche demzufolge gelernt werden muss. Bei einer ausschließlich lexikalischen Verankerung des Genus wäre jedoch die systematische Variation, wie sie bei den auf hybride Nomina verweisenden Pronomina vorliegt, nicht erklärbar. Es dürften demzufolge lediglich genuskongruente Targets verwendet werden, da Sprecherinnen und Sprecher auf das bereits zugewiesene Genus des Controllers zurückgreifen würden.

Dem gegenüber steht die Annahme, dass das Genus motiviert ist und damit außerhalb des Lexikons verortet werden kann. Ähnliche Ansätze wurden bereits durch Dahl (2000), Fahlbusch \& Nübling (2016), Köpcke (1982), Köpcke \& Zubin (1984, 2017), Nübling (2015) sowie Oelkers (1996) verfolgt. Auch die in diesem Beitrag analysierten Korpusdaten lassen den Schluss zu, dass das Genus motiviert ist und in diesem Randbereich des Lexikons „online“ - also erst während des Sprachproduktionsprozesses - zugewiesen wird. Bei der Wahl der passenden Kongruenzform handelt es sich um eine Interaktion von Morphologie, Syntax, Semantik und auch Pragmatik. Die Kongruenz kann in diesem Fall nicht als ausschließlich mechanisch vollzogene Operation gesehen werden, die syntaktische Merkmale wie Numerus und Genus eines Controllers übernimmt und sie auf das Target kopiert, um einen morphosyntaktischen Zusammenhang herzustellen (vgl. Lehmann 1988). Auch Harnisch beschreibt die Pragmatik als Einflussfaktor auf ein Kongruenzphänomen (vgl. Harnisch i. d. B.: 23). Er geht bei der Entstehung von tautologischen Syntagmen wie weibliche Leserinnen ebenfalls nicht von einer rein syntaktischen ablaufenden Kongruenz-Operation aus, sondern nennt als entscheidenden Einfluss die Anpassung an den gendergerechten Sprachgebrauch. ${ }^{8}$

Dass Genus in bestimmte Fällen Bedeutung transportieren kann, zeigen auch andere Studien, wie beispielsweise die Beiträge von Nübling, Busley \& Drenda (2014) und Busley \& Fritzinger (2018) zu neutral markierten Frauenrufnamen (z. B. das Anna). Hier konnte gezeigt werden, dass Genus in einigen Dialekten soziale Beziehungen anzeigen kann. Auch in den hier untersuchten Daten übernimmt das Genus der Targets viel mehr als nur die Funktion von Referenzidentifikation. Es wird angenommen, dass neben der Kongruenzerzeugung mithilfe des Genus des Pronomens auch Bedeutung transportiert wird, insofern,

8 In diesem Zusammenhang wäre sicherlich auch eine Untersuchung des Einflusses von gendergerechtem Sprachgebrauch auf die Pronominalisierung hybrider Nomina spannend. 
als dass das sexuskongruente Target zur Sexualisierung des Kontextes beiträgt. Das Genus übernimmt hier folglich eine konzeptuell-pragmatische Funktion. Diese Hypothese müsste jedoch in einem weiteren Experiment überprüft werden.

\section{Literatur}

Binanzer, Anja, Jana Gamper \& Verena Wecker (i. d. B.): Einleitung.

Birkenes, Magnus Breder, Cleopatra Chroni \& Jürg Fleischer (2014): Genus- und

Sexuskongruenz im Neuhochdeutschen: Ergebnisse einer Korpusuntersuchung zur narrativen Prosa des 17. bis 19. Jahrhunderts. Deutsche Sprache 42, 1-24.

Braun, Friederike \& Geoffrey Haig (2010): When are German 'girls' feminine? How the semantics of age influences the grammar of gender agreement. In Markus Bieswanger, Heiko Motschenbacher \& Susanne Mühleisen (Hrsg.), Language in its socio-cultural context: New explorations in global, medial and gendered uses, 69-84. Frankfurt am Main: Peter Lang.

Brinkmann, Hennig (1962): Die deutsche Sprache. Gestalt und Leistung. Düsseldorf: Schwann. Busley, Simone \& Julia Fritzinger (2018): Em Stefanie sei Mann - Frauen im Neutrum. In

Damaris Nübling \& Stefan Hirschauer (Hrsg.), Namen und Geschlechter. Studien zum onymischen Un/doing gender, 191-212. Berlin, Boston: De Gruyter.

Corbett, Greville G. (2006): Agreement. Cambridge: Cambridge University Press.

Corbett, Greville G. (1991): Gender. Cambridge: Cambridge University Press.

Dahl, Östen (2000): Animacy and the notion of semantic gender. In Barbara Unterbeck, Matti Rissanen, Terttu Nevalainen \& Mirja Saari (Hrsg.), Gender in grammar and cognition. Approaches to gender, 99-116. Berlin, New York: De Gruyter Mouton.

Dal, Ingerid \& Hans-Werner Eroms (2014): Kurze deutsche Syntax auf historischer Grundlage. 4. Aufl. Berlin, Boston: De Gruyter.

Diekmann, Andreas (2009): Empirische Sozialforschung. Grundlagen, Methoden, Anwendungen. 20. Aufl. Reinbek bei Hamburg: Rowohlt.

Dudenredaktion (o. J.): „Mädchen“ auf Duden online. https://www.duden.de/rechtschrei bung/Maedchen (3. August 2018).

Eisenberg, Peter (2013): Der Satz. Grundriss der deutschen Grammatik. 4. Aufl. Stuttgart: Metzler.

Fahlbusch, Fabian \& Damaris Nübling (2016): Genus unter Kontrolle: Referentielles Genus bei Eigennamen - am Beispiel der Autonamen. In Andreas Bittner \& Constanze Spieß (Hrsg.), Formen und Funktionen. Morphosemantik und grammatische Konstruktion, 103-125. Berlin, Boston: De Gruyter.

Fleischer, Jürg (2012): Grammatische und semantische Kongruenz in der Geschichte des Deutschen: eine diachrone Studie zu den Kongruenzformen von ahd. wīb, nhd. Weib. Beiträge zur Geschichte der deutschen Sprache und Literatur 134, 163-203.

Gallmann, Peter (2009): Die flektierbaren Wortarten. In Dudenredaktion (Hrsg.), Duden. Die Grammatik, 145-566. 8. Aufl. Mannheim: Dudenverlag.

Harnisch, Rüdiger (i. d. B.): Von weiblichen Leserinnen und Frauenskispringerinnen. Tautologische Syntagmen auf dem Weg zu festen Konstruktionen. 
Hoberg, Ursula (2004): Grammatik des Deutschen im europäischen Vergleich: Das Genus des Substantivs. Mannheim: Institut für Deutsche Sprache.

Klann-Delius, Gisela (2005): Sprache und Geschlecht. Eine Einführung. Stuttgart, Weimar: J.B. Metzler.

Köpcke, Kaus-Michael (2012): Konkurrenz bei der Genuskongruenz. Überlegungen zum Grammatikunterricht in der Sekundarstufe II. Der Deutschunterricht 1, 36-46.

Köpcke, Klaus-Michael (1982): Untersuchungen zum Genussystem der deutschen Gegenwartssprache. Tübingen: Niemeyer.

Köpcke, Klaus-Michael, Klaus-Uwe Panther \& David A. Zubin (2010): Motivating grammatical and conceptual gender agreement in German. In Hans-Jörg Schmid \& Susanne Handl (Hrsg.), Cognitive foundations of linguistic usage patterns, 171-194. Berlin: De Gruyter.

Köpcke, Klaus-Michael \& David A. Zubin (2017): Genusvariation: Was offenbart sie über die innere Dynamik des Systems? In Marek Konopka \& Angelika Wöllstein (Hrsg.), Grammatische Variation. Empirische Zugänge und theoretische Modellierung, 203-228. Berlin, New York: De Gruyter Mouton.

Köpcke, Klaus-Michael \& David A. Zubin (2009): Genus. In Elke Hentschel \& Petra M. Vogel (Hrsg.), Deutsche Morphologie, 132-154. Berlin: De Gruyter.

Köpcke, Klaus-Michael \& David A. Zubin (2005): Metonymic pathways to neuter-gender human nominals im German. In Klaus Panther \& Linda Thornburg (Hrsg.), Metonomy and pragmatic inferencing, 149-166. Amsterdam: Benjamins.

Köpcke, Klaus-Michael \& David A. Zubin (1984): Sechs Prinzipien für die Genuszuweisung im Deutschen. Ein Beitrag zur natürlichen Klassifikation. Linguistische Berichte 93, 26-50.

Lehmann, Christian (1988): On the function of Agreement. In Michael Barlow \& Charles A. Ferguson (Hrsg.), Agreement in natural language. Approaches, theories, descriptions, 55-65. Stanford: CSLI.

Leiss, Elisabeth (1994): Genus und Sexus. Kritische Anmerkungen zur Sexualisierung von Grammatik. Linguistische Berichte 152, 281-300.

Leiss, Elisabeth (2009): Sprachphilosophie. Berlin: De Gruyter.

Nübling, Damaris (2015): Between feminine and neuter, between semantic and pragmatic gender. Hybrid and neuter female names in German dialects and in Luxembourgish. In Jürg Fleischer, Elisabeth Rieken \& Paul Widmer (Hrsg.), Agreement from a diachronic perspective, 235-265. Berlin, Boston: De Gruyter Mouton.

Nübling, Damaris, Simone Busley \& Juliane Drenda (2014): Dat Anna und s Eva - Neutrale Frauenrufnamen in deutschen Dialekten und im Luxemburgischen zwischen pragmatischer und semantischer Genuszuweisung. Zeitschrift für Dialektologie und Linguistik 80/2, 152-196.

Oelkers, Susanne (1996): ,Der Sprintstar und ihre Freundinnen'. Ein empirischer Beitrag zur Diskussion um das generische Maskulinum. Muttersprache 1, 1-15.

Panther, Klaus-Uwe (2009): Grammatische versus konzeptuelle Kongruenz. Oder: Wann siegt das natürliche Geschlecht? In Rita Brdar-Szabó, Elisabeth Knipf-Komlósi \& Attila Péteri (Hrsg.), An der Grenze zwischen Grammatik und Pragmatik, 67-86. Frankfurt am Main: Peter Lang.

Robinson, Orrin W. (2010): Grimm language. Grammar, gender and genuineness in the fairy tales. Amsterdam, Philadelphia: Benjamins.

Ronneberger-Sibold, Elke (2004): Deutsch. In Geert Booij, Christian Lehmann, Joachim Mugdan \& Stavros Skopeteas (Hrsg.), Morphologie. Morphology. Ein internationales 
Handbuch zur Flexion und Wortbildung. An international handbook on inflection and word-formation. Halbband 2. Volume 2, 1267-1285. Berlin, New York: De Gruyter.

Rosch, Eleanor (1975). Cognitive representations of semantic categories. Journal of

Experimental Psychology: General 104, 192-233.

Rosch, Eleanor (1972): Universals in color naming and memory. Journal of Experimental Psychology 93, 10-20.

Schiller, Anne, Simone Teufel, Christine Stöckert \& Christine Thielen (1999): Guidelines für das Tagging deutscher Textcorpora mit STTS. http://www.sfs.uni-tuebingen.de/resour ces/stts-1999.pdf (25.04.2018).

Thurmair, Maria (2006): Das Model und ihr Prinz. Kongruenz und Texteinbettung bei GenusSexus-Divergenz. Deutsche Sprache 34, 191-220.

Zinsmeister, Heike \& Lothar Lemnitzer (2015): Korpuslinguistik: Eine Einführung. 3. Aufl. Tübingen: Narr.

\section{Korpora}

Directmedia Publishing (Hrsg.) (2005): Deutsche Literatur von Luther bis Tucholsky. Die Grossbibliothek. Berlin: Direct Media Publishing.

Institut für Deutsche Sprache (2016): Deutsches Referenzkorpus / Archiv der Korpora geschriebener Gegenwartssprache 2016-II (Release vom 30.09.2016). Mannheim: Institut für Deutsche Sprache. www.ids-mannheim.de/DeReKo (zuletzt abgerufen am 25.05.2020). 